\title{
REVISIONES
}

\section{La agroforestería como estrategia para la recuperación y conservación de reservas de carbono en bosques de la Amazonía}

\author{
Agroforestry as a strategy for the recovery and conservation of carbon stocks \\ in Amazon forests
}

\author{
Pedro Manuel Villa a,b,ck, Sebastião Venâncio Martins ${ }^{\text {b,d }}$, Luisa Delgado Monsanto ${ }^{\mathrm{c}}$, \\ Silvio Nolasco de Oliveira Neto ${ }^{\mathrm{d}}$, Norman Mota Cancio ${ }^{\mathrm{c}}$ \\ * Autor de correspondencia: ${ }^{a}$ Universidade Federal de Viçosa, Programa de Pós-Graduação em Botânica, \\ CEP 36570000, Viçosa, Minas Gerais, Brasil, tel.: 55-31-83134545, pedro.villa@ufv.br \\ ${ }^{\mathrm{b}}$ Universidade Federal de Viçosa, Departamento de Engenharia Florestal, Laboratório de Restauração Florestal, \\ CEP 36570000, Viçosa, Minas Gerais, Brasil. \\ c Instituto Nacional de Investigaciones Agrícolas, Área de Agroforestería, 7101, Puerto Ayacucho, Amazonas, Venezuela. \\ ${ }^{\mathrm{d}}$ Universidade Federal de Viçosa, Departamento de Engenharia Florestal, CEP: 36570000 , Viçosa, Minas Gerais, Brasil.
}

\begin{abstract}
SUMMARY
Continuous changes in land use in the Amazon in recent years, mainly due to the conversion of forests through agricultural and extractive practices, have resulted in serious alterations in the structure and functioning of ecosystems with impacts at regional and global scales. The replacement of tropical forests by itinerant agricultural systems is one of the main reasons contributing to carbon emissions into the atmosphere and becoming one of the most important environmental impacts. However, there are documented mechanisms for reducing carbon emissions from deforestation and degradation (REDD+), i) controlling the emission reduction sources through conservation and sustainable management of the forest, and ii) recovering and increasing plant biomass as important sinks through forest restoration strategies. In order to increase efficiency in the recovery and conservation of carbon in vulnerable deforested and degraded forest areas of the Amazon, our objective was to develop an analysis of the potential environmental impacts of agroforestry as a REDD+ alternative, through i) rehabilitation of degraded areas subjected to successive fire and crop cycles, ii) passive restoration of secondary forests, and iii) implementation of improved fallows managing agroforestry species. Agroforestry is an important alternative to recover and conserve carbon stocks through REDD+ activities against the shifting cultivation in the Amazon.
\end{abstract}

Key words: deforestation, forest degradation, improved fallow, passive restoration, rehabilitation.

\section{RESUMEN}

Durante los últimos años los continuos cambios en el uso de la tierra en la Amazonía, principalmente a través de la conversión de bosques por prácticas agropecuarias y de extracción, han generado graves transformaciones en la estructura y funcionamiento de los ecosistemas con impactos a escala regional y global. La deforestación para establecer sistemas agrícolas itinerantes es uno de los principales factores que contribuyen con las emisiones de carbono a la atmósfera como uno de los servicios de mayor repercusión ambiental. Sin embargo, existen reconocidos mecanismos de reducción de emisiones de carbono por deforestación y degradación (REDD+), i) bien sea controlando la reducción de emisiones de las fuentes a través de la conservación y manejo sustentable de los bosques, y ii) recuperando e incrementando la biomasa vegetal como importantes sumideros a través de estrategias de rehabilitación y restauración forestal. Con la finalidad de incrementar la eficiencia en la recuperación y conservación de carbono en áreas vulnerables de deforestación y degradación forestal en la Amazonía, se propone como objetivo desarrollar un análisis de los impactos ambientales potenciales de la Agroforestería como alternativa REDD+, a través de i) la rehabilitación de áreas degradadas sometidas a ciclos sucesivos de fuego y cultivos, ii) la restauración pasiva de bosques secundarios, e iii) implementación de barbechos mejorados con el manejo de especies agroforestales. La agroforestería representa una importante alternativa para recuperar y conservar reservas de carbono a través de las principales actividades REDD+ frente a la presión generada por la agricultura itinerante en la Amazonía.

Palabras clave: deforestación, degradación forestal, barbecho mejorado, restauración pasiva, rehabilitación. 


\section{INTRODUCCIÓN}

La degradación de los bosques tropicales tiene consecuencias negativas significativas sobre diferentes componentes estructurales y funcionales de los ecosistemas, por lo tanto, también en su capacidad de proporcionar bienes y servicios ambientales con impactos regionales y globales; como es el caso de las reservas de carbono en el suelo y biomasa vegetal, regulación de procesos climáticos, y mantenimiento de la biodiversidad (Thompson et al. 2012, 2013). Por esta razón, actualmente los esfuerzos de investigación, ajustes de políticas ambientales y estrategias de gestión a nivel mundial, están dedicando especial atención a la importancia de la conservación y manejo de los bosques tropicales con el propósito de reducir las emisiones de carbono por deforestación y degradación forestal, y mantener un uso sustentable de la tierra para fines agrícolas. En este contexto, existe una particular preocupación por la cuenca amazónica como una de las mayores extensiones de bosques tropicales con mayor capacidad de secuestro y almacenamiento de carbono a nivel mundial (Malhi et al. 2006, Pan et al. 2011, Saatchi et al. 2011, Aragão et al. 2014), debido a que presentan alarmantes tasas de deforestación actual y acumulada, siendo una de las mayores en comparación a otras regiones tropicales (Margulis 2004, Laurance et al. 2004, Cerri et al. 2007, Nepstad et al. 2009, INPE 2013).

Se estima que los bosques tropicales pueden almacenar aproximadamente 247 Pg de carbono en la biomasa aérea y subterránea (Saatchi et al. 2011), mientras que existen otras estimaciones actuales de carbono que están en el orden de $471 \pm 93 \mathrm{Pg}$, almacenado en la biomasa y otros compartimientos como el suelo, necromasa y hojarasca acumulada (Pan et al. 2011). No obstante, debido a los continuos cambios en el uso de la tierra, principalmente a causa de la deforestación y quema para la expansión de la frontera agrícola, una gran parte del carbono almacenado en la biomasa se libera a la atmósfera como dióxido de carbono, en consecuencia el carbono almacenado en los suelos, en el mantillo de hojarasca y materia orgánica, también puede convertirse en una fuente de emisiones debido al incremento de la respiración derivada de la descomposición (Lal 2008, Thompson et al. 2012).

La Amazonía ha sido un importante sumidero de carbono durante las últimas tres décadas, pudiendo llegar a almacenar aproximadamente $93 \mathrm{Pg}$ en la biomasa aérea y subterránea; además existen resultados consistentes sobre su alto potencial para el secuestro de carbono como uno de los servicios ambientales de mayor repercusión global (Malhi et al. 2006, Cerri et al. 2007, Saatchi et al. 2011), siendo Brasil el país que presenta la mayor extensión de área, con más del $60 \%$ de toda la cuenca, convirtiéndolo en una región estratégica para la conservación del carbono y mitigación al cambio climático (Soares-Filho et al. 2006, Soares-Filho et al. 2010, INPE 2013). Sin embargo, un estudio reciente revela que los bosques de la Amazonía están presentando una tendencia a incrementar la tasa de mortalidad de árboles repercutiendo sobre la capacidad de almacenamiento de carbono, por eso también se presume que los impactos del cambio climático pueden tener un efecto preponderante. Así mismo, se conoce que las regiones tropicales han contribuido significativamente como sumidero mundial de carbono entre los años 1980 y 1990, mientras que los resultados actuales muestran que el carbono neto total almacenado en la biomasa de bosques intactos de la Amazonía ha disminuido en un $30 \%$, desde $0,54 \mathrm{Pg}^{2}$ año $^{-1}$ de carbono en la década de 1990 , hasta $0,38 \mathrm{Pg}_{\text {año }}{ }^{-1}$ en la década de 2000 (Brienen et al. 2015).

En las últimas dos décadas, las emisiones netas de carbono derivadas de la deforestación y la degradación tropical eran casi iguales a las emisiones totales procedentes de los cambios en el uso de la tierra a escala global $(1,11 \mathrm{Pg}$ año ${ }^{-1}$ de carbono), porque los efectos de estos cambios fueron más o menos equilibrados en zonas tropicales, influyendo en el papel que desempeñan los bosques tropicales como sumideros de carbono a largo plazo (Pan et al. 2011). La agricultura de tala y quema también ha contribuido de manera significativa a las emisiones globales de carbono en los países tropicales (Thompson et al. 2012), pero aún no existen suficientes estimaciones razonables de las emisiones causadas por este tipo de agricultura itinerante a nivel de la cuenca amazónica, y que actualmente representa un modelo de uso de la tierra no sustentable. A pesar de la reducción de hasta un $80 \%$ de la deforestación en la cuenca amazónica entre los años 2005 y 2013 (INPE, 2013), donde Brasil sigue siendo responsable de aproximadamente el $40 \%$ de los bosques lluviosos tropicales que quedan en el mundo, teniendo un papel vital en el mantenimiento de reservas de carbono terrestre (Laurance et al. 2001), aún prevalece una constante degradación forestal debido a la tala selectiva, deforestación para la agricultura, e incendios forestales (Nobre y Borma 2009, Boucher et al. 2013, INPE 2013, Aragão et al. 2014).

Varios investigadores han analizado diferentes escenarios ambientales frente a los continuos cambios en el uso de la tierra causados por la deforestación en la cuenca amazónica, observando que dichas transformaciones en los ecosistemas repercuten en la pérdida significativa de sumideros de carbono, además de considerables impactos negativos sobre el balance a escala global (Laurance et al. 2001, Laurance et al. 2004, Aragão et al. 2014, Gatti et al. 2014). La deforestación en esta región es causada principalmente para la ampliación de áreas cultivadas e incremento de los rendimientos de producción agrícola para cubrir las demandas a escala familiar y mercados locales, así como para mercados nacionales e internacionales (SoaresFilho et al. 2006, Porro et al. 2012, Boucher et al. 2013). Además de las áreas destinadas para cultivos extensivos de soya y la ganadería que ocupa más del $85 \%$ de las tierras agrícolas en la región (Soares-Filho et al. 2006, Boucher et al. 2013), el tipo de agricultura más practicada se ha caracterizado por ser itinerante, tradicionalmente conoci- 
do en diferentes países que conforman la cuenca, como conuco, coivara o chakra, y del cual se desconoce la proporción de área total utilizada en toda la cuenca. Este modelo agrícola consiste en la deforestación de 0,5 a 1,5 ha de bosques primarios o secundarios como parte de la preparación del terreno para el establecimiento de cultivos durante un corto período de tiempo, generalmente de dos a tres años hasta su abandono cuando disminuye la fertilidad de los suelos (D'Oliveira et al. 2011, Arroyo-Kalin 2012). Por este motivo, la conservación y manejo sustentable de los bosques en la Amazonía también ha cobrado más importancia durante la última década, frente a la necesidad de prevenir y mitigar el efecto de la acelerada deforestación y degradación forestal causada por diferentes cambios en el uso de la tierra y efectos del cambio climático, principalmente para reducir las emisiones de carbono a la atmósfera (Soares-Filho et al. 2006, Nobre y Borma 2009, Nepstad et al. 2009, Soares-Filho et al. 2010, Porro et al. 2012, Aragão et al. 2014).

Por estos motivos, la conservación de los bosques amazónicos depende en gran parte de un conjunto de estrategias y acciones orientadas a optimizar el uso y manejo de bienes y servicios ecosistémicos, que simultáneamente garanticen la conservación de reservas de carbono, primordialmente a través de la agricultura. En esa búsqueda de un equilibrio entre desarrollo y conservación, la agroforestería ha sido implementada como una propuesta de agricultura sustentable basada en conocimientos tradicionales e innovaciones tecnológicas, incluso con destacadas experiencias exitosas en comunidades indígenas y rurales (Porro et al. 2012, Tremblay et al. 2015). La agroforestería como un tipo de manejo de bosque, también es conocida como un modelo de agricultura climática inteligente, porque puede contribuir a incrementar la productividad agrícola basada en la biodiversidad, generando medios de vida sustentables en la misma medida que se controla la deforestación y degradación forestal, como las dos principales actividades para reducir las emisiones de carbono a la atmósfera (REDD). Por otra parte, también es considerada como una estrategia que comprende tres actividades complementarias para la conservación, manejo sustentable e incremento de las reservas de carbono en los bosques (REDD+) a través de la rehabilitación de áreas degradadas (Nair et al. 2010, Kapos et al. 2012). En todo caso, a pesar de que la discusión sobre el impacto derivado de los diferentes tipos de manejo de bosques asociados a las cinco actividades REDD + no es un asunto nuevo, y que la agroforestería es una práctica antigua y ampliamente conocida a través de numerosas experiencias de campo y publicaciones de diferentes índole, desafortunadamente no existe una propuesta integral que analice simultáneamente las potencialidades y relaciones existentes para la conservación e incremento de reservas de carbono como estrategia alternativa de mitigación ambiental frente a la deforestación para el establecimiento de sistemas agrícolas itinerantes que traen como consecuencia la formación de paisajes forestales fragmentados y con diferentes niveles de degradación, así como la emisión de carbono a la atmósfera.

Por esta razón, se presenta una revisión descriptiva y analítica donde se contextualiza sistemáticamente aspectos teóricos y conceptuales sobre algunos impactos ambientales potenciales de la agroforestería sobre el manejo de bosques en la Amazonía, como alternativa para incrementar la eficiencia en la conservación y recuperación de carbono en áreas vulnerables de deforestación y degradación forestal a causa de la agricultura itinerante. En la primera sección de este trabajo, se justifica como la agroforestería representa un modelo que integra diferentes actividades REDD+ de agenda mundial, y posteriormente se describen dichas estrategias basadas en métodos de restauración forestal. De este modo, se discute cómo la agroforestería puede representar un modelo de gestión de referencia a escala de toda la cuenca, aun cuando se reconocen que otros tipos de uso de la tierra siguen siendo las causas de mayor impacto de deforestación y degradación. Esta revisión está fundamentada en los criterios actuales considerados por la Unión Internacional de Organizaciones de Investigación Forestal (International Union of Forest Research Organizations, IUFRO) sobre la relación existente entre bosques y carbono como aspecto clave para alcanzar los objetivos REDD+ a escala global.

\section{LA AGROFORESTERÍA COMO ESTRATEGIA REDD+}

Es bien conocido que el carbono es un elemento fundamental en diferentes procesos biológicos, ecológicos y biogeoquímicos desde una escala de organismos vivos hasta una escala global, el cual puede ser almacenado y transferido constantemente entre reservorios distribuidos en la biósfera, atmósfera, hidrósfera y litósfera. En este sentido, los océanos representan el compartimiento que almacena la mayor cantidad, con aproximadamente $38.400 \mathrm{Pg}$ de carbono, mientras que las reservas orgánicas del suelo oscilan entre $1.550 \mathrm{Pg}$, la atmósfera con $760 \mathrm{Pg}$, y el componente biótico con $560 \mathrm{Pg}$. El ciclo biológico consiste en el intercambio de gases entre la biomasa viva y la atmósfera, donde la absorción del $\mathrm{CO}_{2}$ por las plantas ocurre a través de la fotosíntesis, y cerca del $50 \%$ del total absorbido es liberado inmediatamente por la respiración autotrófica; así también, la respiración heterotrófica que ocurre en el suelo durante el proceso de descomposición representa una fuente de retorno a la atmósfera (Lal 2008, Ciais et al. 2013).

Lal (2008) y Ciais et al. (2013) mencionan que las actividades humanas producen una emisión anual aproximada de 8 Pg de carbono, de los cuales 6,4 Pg provienen de combustibles fósiles, y 1,6 Pg de la deforestación como parte de los cambios en el uso de la tierra, siendo la segunda fuente global más importante de emisiones de $\mathrm{CO}_{2}$ a la atmósfera. Los bosques con sus suelos contienen hasta tres veces la cantidad de carbono contenida actualmente en la 
atmósfera, y procesan anualmente a través de la fotosíntesis y la respiración una cantidad equivalente de 15 a $20 \%$ de ese total. Estos procesos biogeoquímicos son fundamentales para mantener los patrones climáticos globales y regionales, evidenciando la importancia de los bosques para el mantenimiento de flujos y reservorios. Frente a este escenario, es relevante indicar que la transformación de los bosques tropicales debido a la deforestación ha sido un proceso continuo a partir del periodo pre agrícola hace unos 8.000 años, estimándose una pérdida del $50 \%$ de la cobertura forestal original del planeta principalmente a causa de la agricultura, siendo la mayor parte durante las últimas tres décadas. Hasta hace un poco más de una década se estimó que cerca de un $40 \%$ de los bosques del mundo se encontraban poco intervenidos, pero que de igual forma eran áreas vulnerables de deforestación para la agricultura (Bryant et al. 1997, Roosevelt 2013). En el caso de los bosques de la Amazonía, estudios arqueológicos demuestran que han estado sometidos a la deforestación y uso del fuego para el establecimiento de sistemas agrícolas itinerantes durante toda la era antropocénica (Arroyo-Kalin et al. 2012, Roosevelt 2013).

Por otra parte, es importante destacar que los bosques lluviosos tropicales poseen una considerable capacidad de almacenamiento de carbono, que oscila entre 100 y $300 \mathrm{Mg}$ $\mathrm{ha}^{-1}$, y la producción de biomasa puede variar entre $70 \mathrm{y}$ $400 \mathrm{Mg} \mathrm{ha}^{-1}$ (Baker et al. 2004, Saatchi et al. 2011), mientras que los bosques de la Amazonía como mayor reserva de carbono del planeta puede presentar una capacidad aproximada de almacenamiento de $200 \mathrm{Mg} \mathrm{ha}^{-1}$; aunque todavía se conoce muy poco sobre el nivel de variación a causa de los cambios actuales en el uso de la tierra, variación de los patrones climáticos, y efecto de los incendios forestales (Malhi et al. 2006, Pan et al. 2011, Saatchi et al. 2011, Aragão et al. 2014, Gatti et al. 2014). Otras investigaciones explican que durante las últimas tres décadas ha estado ocurriendo un incremento en la biomasa vegetal de los bosques húmedos neotropicales, observándose el mismo patrón en diferentes bosques amazónicos, por eso se discute que la principal causa ha sido la elevada concentración de carbono en la atmósfera que estimula la producción primaria neta (Baker et al. 2004, Malhi et al. 2006, Pan et al. 2011, Saatchi et al. 2011); aunque resultados de una investigación actual indican que los sumideros de carbono están declinando debido a una disminución de la tasa de cambio de biomasa neta y un aumento de las tasas de mortalidad cuando se analizan a una escala de tiempo de largo plazo, representando un incremento de $30 \%$ de las reservas de necromasa desde 1983 hasta 2012 (Brienen et al. 2015). Bajo estos escenarios actuales y futuros, queda evidente de que las diferentes estrategias de conservación y manejo sostenible de los bosques de la Amazonía, no son suficientes alternativas para la conservación, recuperación e incremento de las reservas de carbono, y que deben implementarse actividades REDD + complementarias como medios de mitigación ambiental. Frente a esta perspectiva, se destacan las ventajas de los sistemas agroforestales para la recuperación de áreas forestales degradadas e incremento de las reservas alternativas de carbono a corto plazo, a partir de 10 hasta 30 años, e incluso también podría ser a largo plazo cuando se mantienen ciclos agroforestales sucesivos en la misma unidad de producción.

Este incremento significativo de emisiones de carbono a la atmósfera, han generado cambios importantes en el patrón de acumulación y dinámica del mismo a escala global, lo que simultáneamente ha influido sobre alteraciones en el clima a un ritmo alarmante (Ciais et al. 2013). A pesar de atravesar este escenario ambiental, son bien conocidas dos estrategias propuestas para mitigar el incremento de carbono en la atmósfera, i) controlando la reducción de emisiones a través del manejo de las fuentes, e ii) incrementando la biomasa vegetal como sumideros a través de prácticas de forestación, reforestación, y rehabilitación, siendo esta última relevante con el establecimiento de sistemas agroforestales. De este modo, con la agroforestería se pueden conservar bosques primarios con mayores reservorios de carbono, limitando la demanda de áreas forestales para el establecimiento de nuevos ciclos de cultivos itinerantes, y en consecuencia reduciendo la deforestación y degradación forestal (Kapos et al. 2012), o través del manejo de bosques secundarios durante las primeras etapas de sucesión, después que es abandonado un sistema agrícola itinerante, también conocidos como barbechos mejorados o sistemas agroforestales sucesionales (Vieira et al. 2009, Kapos et al. 2012).

Es conveniente resaltar que en la actualidad los sistemas agroforestales representan el modelo agrícola más apropiado para la recuperación y conservación de bosques tropicales (Vieira et al. 2009, Kapos et al. 2012), por ser un sistema de uso de la tierra donde se cultivan especies agrícolas y arbóreas en la misma unidad de producción durante un prologando periodo de tiempo, con arreglos espaciales y temporales específicos en función de las características biológicas y ecológicas de cada especie, y de las condiciones ambientales de cada localidad. También es necesario señalar que la descripción funcional de los sistemas agroforestales aún se encuentra en constante evaluación para comprender mejor la relación existente entre producción y conservación de bosques tropicales. Además, se destaca que más allá de la cosecha de productos agroforestales y conservación de reservas de carbono, estos sistemas también podrían generar otros tipos de servicios ambientales, como la conservación de suelos para aumentar la productividad y mitigar los procesos erosivos, aumentar la biodiversidad de organismos descomponedores y mantener los niveles de fertilidad.

\section{CONSERVACIÓN DE RESERVAS DE CARBONO}

Los bosques son los ecosistemas que secuestran y almacenan mayor cantidad de carbono en la biomasa vegetal y en el suelo, por lo que es bien reconocido tienen una fun- 
ción importante en el intercambio de carbono entre la biosfera y la atmósfera, siendo los bosques de la Amazonía los más importantes desde el punto de vista de conservación de reservas de carbono (Lal 2008, Pan et al. 2011, Saatchi et al. 2011). Buena parte de las reservas de carbono en la región, hasta un $50 \%$ de almacenamiento y productividad, depende de los árboles con mayor abundancia que está fuertemente sesgada hacia relativamente pocas especies hiperdominantes, representando apenas $1 \%$ del total de especies (Fauset et al. 2015). Sin embargo, se ha determinado que las tasas de deforestación en el Amazonas brasileño han sido una de las más rápidas del mundo, especialmente entre los bosques tropicales, con un promedio de pérdida de $17.486 \mathrm{~km}^{2}$ año-1 (Espindola et al. 2012). Por lo tanto, comprendiendo los principales patrones y procesos determinantes en la estructura y funcionamiento de los bosques amazónicos, principalmente los relacionados con la dinámica del carbono en el mantenimiento de la productividad y biodiversidad, será posible tener mejores aproximaciones en la estimación de los impactos generados por la agroforestería como estrategia REDD+ frente a la deforestación para el establecimiento de sistemas agrícolas itinerantes. Además de la agroforestería, se reconoce que existen diferentes tipos de acciones de manejo de bosques que también pueden contribuir con las cinco actividades REDD+, destacándose la importancia del i) manejo del fuego, ii) la reducción de la tala selectiva, iii) la aplicación de métodos de restauración y reforestación, iv) así como la ordenación ambiental para delimitar dichas acciones.

Frente a este contexto ambiental, los patrones espaciales y temporales de pérdida de bosques en la Amazonía también han sido variables y heterogéneos que dificultan su entendimiento, aunque es evidente que las áreas con mayor tasa de deforestación ocurren en Brasil, entre el este y sur de la cuenca, en los estados de Pará, Mato-Grosso y Rondonia, considerados también como zonas calientes de deforestación (Laurance et al. 2001, Espindola et al. 2012, Porro et al. 2012, Diniz et al. 2013, Verburg et al. 2014) con poca potencialidad para la conservación de reservas de carbono. En la Amazonía brasileña, las tasas anuales de deforestación estimadas oscilan entre 1,1 hasta 2,9 millones de hectáreas, mientras que el área total deforestada ha alcanzado aproximadamente 85 millones de hectáreas, aproximadamente el $14 \%$ de la superficie total (INPE 2013). Aunque existe un escenario negativo, también se cuenta con estrategias esperanzadoras para reducir la deforestación, y con los esfuerzos recientes se ha logrado ampliar la red de áreas protegidas de 1,26 a 1,82 millones de kilómetros cuadrados adicionales, que contienen el $51 \%$ de área restante de bosque en la región (Nepstad et al. 2009, Soares-Filho et al. 2010, INPE 2013). A pesar de todo, la reducción de la deforestación en los últimos años ha tenido lugar frente a las continuas presiones en el uso de la tierra, especialmente por los impactos causados por la ampliación de las plantaciones de soya y áreas ganaderas de Brasil como actividades de alta rentabilidad económica
(Soares-Filho et al. 2006, Boucher et al. 2013, Verburg et al. 2014).

Una primera aproximación consistente sobre algunos criterios ambientales que permiten evaluar la dinámica de la deforestación de algunas regiones de la Amazonía ha sido analizando la variabilidad climática, como el efecto que tienen las bajas precipitaciones, las severas y prolongadas estaciones de déficit hídrico, y suelos con baja fertilidad, principalmente sobre la vulnerabilidad de los bosques deciduos y semideciduos de la cuenca (Nobre y Borma 2009, Aragão et al. 2014, Gatti et al. 2014), que muy probablemente repercute en la productividad en términos de ganancia de carbono y en la capacidad de regeneración después que ocurre una perturbación. Así mismo, los incendios antropogénicos se están convirtiendo en una causa cada vez más común en la degradación forestal, especialmente en las zonas con mayor deforestación y fragmentación (Nobre y Borma 2009, Aragão et al. 2014). En consecuencia, se presume que el sur y este de la Amazonía, con dominancia de bosques estacionales, seguiría siendo la región más vulnerable a la degradación forestal por su alta sensibilidad a la incidencia de incendios antropogénicos y condiciones climáticas extremas, y en consecuencia una menor capacidad para la recuperación y conservación de reservas de carbono.

Es evidente que para controlar la deforestación causada por diferentes actividades de envergadura dentro de la cuenca amazónica, se necesitará de mayor esfuerzo en el ajuste, conciliación y aplicación rigurosa de políticas agrarias de desarrollo rural y políticas de conservación ambiental, especialmente por el impacto causado por la ganadería extensiva, plantaciones de soya, la minería, y tala selectiva para fines industriales. Estas tendencias actuales de uso de la tierra, con probabilidades de expansión en la Amazonía, permiten presumir que se está gestando un círculo vicioso de degradación forestal con autoperpetuantes consecuencias ecológicas que afectaría la resiliencia de los ecosistemas y capacidad de secuestrar y almacenar carbono. Sin embargo, la agroforestería como estrategia REDD+ frente a la situación actual, puede contribuir a conservar y recuperar las reservas de carbono, por lo menos mitigando la presión por deforestación de nuevas áreas para fines agrícolas, a través de la implementación de métodos comprobados de rehabilitación de áreas forestales degradadas en relación a los gradientes climáticos y potencialidades ecológicas de las especies. Estas actividades REDD+ deben ser planificadas desde una escala local hasta una regional, bajo diferentes escenarios actuales y futuros de cambios en el uso de la tierra que permitan ordenar y clasificar áreas potenciales para la conservación y manejo sustentable de bosques, así como de acuerdo a la capacidad de resiliencia y vulnerabilidad de degradación. Además de la importancia de integrar las actividades REDD+ en todos los escenarios, también se destaca la relevancia potencial que podrían tener las especies hiperdominantes para la restauración y rehabilitación forestal. 


\section{RECUPERACIÓN DE RESERVAS DE CARBONO}

La recuperación de las reservas de carbono en una matriz de paisaje forestal de la Amazonía, donde existen áreas con diferentes estados de conservación y manejo para el establecimiento de sistemas agrícolas itinerantes, es posible a través de la implementación de diferentes actividades REDD+. De acuerdo a este patrón generalizado de uso de la tierra en la cuenca, y según las potencialidades de la agroforestería a escala regional, en esta revisión se presentan tres acciones para recuperar e incrementar las reservas de carbono en los diferentes tipos de bosques. En este sentido, se propone i) la rehabilitación de áreas degradadas sometidas a ciclos sucesivos de uso del fuego y cultivos, y con poco tiempo de barbecho, que afecta la capacidad de regeneración del bosque. Con la rehabilitación de áreas forestales degradadas también se contribuye con ii) la restauración pasiva evitando el uso continuo de bosques secundarios durante diferentes etapas de sucesión después de un ciclo de cultivo itinerante, y evitando la deforestación de bosques primarios. En este caso, también se podría implementar un manejo sustentable de los bosques secundarios a través de iii) barbechos mejorados, aumentando la incorporación y densidad de siembra de especies arbóreas agroforestales de ciclo largo durante la sucesión controlada, después de un sistema agrícola itinerante.

Rehabilitación de áreas forestales degradadas. Existen reportes generalizados de que el tiempo y capacidad de recuperación de carbono de los bosques después de una perturbación, dependen de la escala, intensidad y las formas de degradación (Thompson et al. 2012, 2013), como ha sido descrito para bosques de Borneo bajo una agricultura itinerante por más de 200 años, y donde la acumulación de biomasa fue significativamente menor en los sitios sometidos a seis o más ciclos de cultivo, debido a una pérdida de bancos de semillas y capacidad de rebrote de especies (Lawrence 2004). Pero en todos los casos dependerá del efecto multifactorial entre la resiliencia de los ecosistemas y métodos de rehabilitación agroforestal implementados. En la cuenca Amazónica, el fuego ha sido utilizado durante muchas generaciones como un medio para el manejo de la tierra con fines agrícolas (Arroyo-Kalin 2012, Roosevelt 2013), del mismo modo ha sido identificado como un agente físico que ha estado influyendo en varios procesos ambientales responsables de la degradación forestal, aunque se desconoce la magnitud de sus consecuencias a escala regional. También se conoce que el fuego tiene un fuerte efecto negativo sobre la acumulación de carbono en bosques secundarios, debido a que con cinco o más ciclos de fuego en una misma área, se puede reducir significativamente en más del $50 \%$ del total, además de acelerar la degradación forestal (Zarin et al. 2005, Thompson et al. 2012, 2103). Por lo tanto, es de esperarse que el proceso de rehabilitación agroforestal pueda ser más complejo y difícil en áreas sometidas a excesivos e intensos ciclos de fuego, donde los bosques secundarios disminuyen su capacidad de recuperar su carbono original dentro del intervalo promedio de retorno. Esto también ocurre por la continua mortalidad post-incendio y baja fertilidad de los suelos, además de la reducción de las tasas de acumulación de biomasa por la dominancia de especies ruderales herbáceas y arbustivas con baja densidad de la madera.

En cualquier escenario, se espera que los sistemas agroforestales establecidos en áreas forestales degradadas puedan llegar a secuestrar y almacenar mayores cantidades de carbono en la biomasa vegetal y suelos en comparación con los sistemas agrícolas itinerantes sin árboles y de corto periodo de tiempo. Por eso, la plantación de árboles en áreas degradadas podría ser relativamente eficiente comparada con otras estrategias de uso del bosque, debido a que producen mayor cantidad de hojarasca que mejora paulatinamente la fertilidad de los suelos y favorece la acumulación de carbono a corto plazo (Nair et al. 2010, Kapos et al. 2012). Con este modelo es posible conservar cantidades importantes de carbono en la biomasa mientras se producen alimentos y se conserva la biodiversidad local durante largos periodos de tiempo bajo ciclos sucesivos de cultivos perennes en la misma unidad de producción, donde cada ciclo agroforestal puede comprender entre dos y tres décadas. En ese aspecto, se estima que el establecimiento de sistemas agroforestales a escala global puede capturar cerca de 38 billones de megagramos de carbono, lo que equivaldría aproximadamente a $15 \%$ del total de las emisiones fósiles del mismo período, con una capacidad de almacenamiento de carbono que oscila entre 12 y $200 \mathrm{Mg}$ $\mathrm{ha}^{-1}$, y aproximadamente $95 \mathrm{Mg} \mathrm{ha}^{-1}$ en zonas tropicales (Albrecht y Kandji 2003, Nair et al. 2010). Además, Albrecht y Kandji (2003) considerando el carbono en la biomasa aérea y en el suelo, y estandarizando los valores a 50 años de rotación, indican que los sistemas agroforestales podrían acumular entre 1,1 y 2,2 Pg de carbono en todo el mundo, con lo que podría reducir la tasa de acumulación del mismo en la atmósfera. Así mismo, las diferentes prácticas silvícolas como la selección, asociación y densidad de plantación de las especies, deben ser criterios técnicos determinantes en los procesos asociados con la captura de carbono en sistemas agroforestales, lo que debe ser considerado para las diferentes condiciones ambientales y ecológicas de la cuenca amazónica.

Restauración pasiva. Es bien conocido que existe un impacto negativo significativo de la deforestación y el fuego sobre la capacidad de regeneración del bosque secundario en la Amazonía. No obstante, es importante resaltar que después del primer ciclo de uso de la tierra las tasas de acumulación de carbono en la biomasa aérea suelen ser mayores a corto plazo durante las dos primeras décadas de sucesión debido a una mayor demanda para el rápido crecimiento, aunque a largo plazo después de 30 años tiende a estabilizarse hasta alcanzar paulatinamente niveles de un bosque primario (Feldpausch et al. 2004). Además, la 
productividad primaria neta en bosques secundarios puede llegar a ser de tres a cinco veces mayor que la de los bosques primarios, aunque las reservas de carbono en los bosques primarios son mucho mayores. Por esta razón, los bosques secundarios representan un destacado sumidero de carbono a escala mundial que compensa parcialmente las emisiones causadas por las altas tasas de deforestación en las mismas regiones tropicales, incluyendo la cuenca amazónica (Luyssaert et al. 2008, Feldpausch et al. 2004). Cabe destacar que, hasta ahora no existen estudios que consideren el efecto sinérgico de la intensidad, frecuencia y duración de la agricultura itinerante sobre los procesos biogeoquímicos de bosques amazónicos, especialmente sobre la dinámica del carbono; aunque es razonable presumir como patrón general, que los repetidos ciclos de tala y quema están afectando la capacidad de recuperación de la biomasa y por lo tanto repercute en la pérdida progresiva de carbono.

La dinámica de sucesión natural del bosque secundario, es también conocida como una estrategia de restauración forestal, que consiste en comenzar a conservar permanentemente áreas que fueron previamente deforestadas. Sin embargo, una vez que los bosques alcanzan la madurez cuando la acumulación de carbono comienza a estabilizarse, es posible que haya una disminución de este importante sumidero al punto que podría mantener un balance neutro entre la acumulación y pérdida de biomasa, incluso no se descarta un escenario futuro donde el balance neto de biomasa presente valores significativamente negativos si no se colocan en práctica diferentes acciones sinérgicas de mitigación ambiental a través de las actividades REDD+. En este sentido, el balance neto de carbono de los bosques amazónicos depende de la contribución relativa de los sumideros actuales en proceso de regeneración, y de las pérdidas por deforestación. Por esta razón, estimando los niveles de déficit de sumideros que podrían generar diferentes escenarios de desequilibrio, sería posible ejecutar acciones integrales REDD+ en función de la cantidad necesaria de carbono que debería ser recuperado y almacenado en sumideros potenciales y alternativos, como por ejemplo a través de diferentes modelos agroforestales.

D'Oliveira et al. (2011) evalúan la regeneración de bosques amazónicos después de diferentes severidades de quema y concluyeron que los resultados fueron consistentes con otros estudios realizados en la misma región, donde los distintos regímenes de severidad pueden producir fuertes diferencias en la regeneración natural, en la estructura, composición, y acumulación de biomasa. Generalmente, la práctica de la restauración pasiva ocurrirá en áreas que fueron usadas anteriormente para el establecimiento de sistemas agrícolas, y donde el uso del fuego puede generar cambios significativos en la dinámica de sucesión vegetal. Así mismo, es importante tener presente que el uso del fuego es sólo una de las principales prácticas de manejo de la agricultura itinerante, por lo que el efecto ponderando de todas las posibles prácticas implementadas previamente causaría un mayor impacto sobre los procesos de restauración pasiva. Finalmente, es importante señalar que la recuperación de reservas de carbono también podría comenzar desde los mismos sistemas agrícolas itinerantes recientemente establecidos, a través de la agroforestería sucesional, bajo la condición de que sea el último ciclo de manejo tradicional de tala y quema.

Restauración agrosucesional. Existe una limitada investigación sobre el desarrollo de sistemas agroforestales regenerativos análogos (SAFRA) y barbechos mejorados en la Amazonía como alternativas de manejo de los bosques secundarios con relación a la dinámica de ganancia de carbono, en particular sobre la fenología reproductiva de las especies, y por lo tanto en la sincronización y arreglos espaciales. No obstante, existen otras razones importantes y conocidas para su adopción que en muchas regiones de la cuenca puede iniciar a partir de un sistema agrícola itinerante, o también después del abandono durante la etapa de barbecho cuando inicia la sucesión de especies de árboles nativos, e incluso en cualquiera de las etapas durante la dinámica conuco-barbecho a través de una restauración planificada. En estos sistemas es posible establecer diferentes especies agroforestales para simular la dinámica de la sucesión natural del bosque secundario; es decir, considerando la cronosecuencia de establecimiento de acuerdo con los ciclos de vida de las mismas y controlando el crecimiento de las especies nativas, por lo que durante la primera etapa se aprovecha la productividad de los cultivos anuales combinados con arbustos de ciclo corto, mientras se establecen y desarrollan las plántulas de frutales de ciclo largo.

Los barbechos mejorados, como modelo de restauración agrosucesional, comienzan cuando nuevos cultivos de ciclo medio y largo, generalmente frutales, son plantados durante la etapa inicial de sucesión natural de especies nativas, a veces justo después de la cosecha de especies anuales y bianuales cuando el sistema agrícola es abandonado. Los sistemas de barbechos mejorados pueden ser productivos por un rango de tiempo comprendido entre $5 \mathrm{y}$ 20 años de cosecha (Vieira et al. 2009). Estos modelos agroforestales son a veces más productivos que sistemas de sucesión sin especies agroforestales conocidos como barbechos improductivos, presentando mayor acumulación de hojarasca en el suelo, y mayor almacenamiento de carbono en la biomasa aérea. Según diferentes experiencias de campo en Amazonas, se ha observado que en las comunidades indígenas los sistemas de cultivo generalmente están asociados con especies nativas de los bosques creciendo simultáneamente sin una disposición espacial sistemática, pero en términos de producción puede ser mayor que los monocultivos o bosques secundarios degradados.

En el caso de la Amazonía brasileña, la agroforestería es fundamental para mantener un desarrollo sustentable en áreas de preservación permanente y áreas de reserva legal a través de la creación de áreas productivas en pequeñas 
propiedades indígenas o rurales (Schroth et al. 2006). En estas unidades de conservación la agroforestería tiene la ventaja de contar con una alta biodiversidad y recursos locales, en la misma medida que se protegen otras áreas de bosques primarios o secundarios con diferentes etapas de sucesión. Por lo tanto, de conformidad con las normas legales y criterios técnicos y ambientales de cada tipo de área protegida, se propone el modelo SAFRA para áreas de preservación permanente, que también son conocidos con otros nombres como forest garden o barbechos mejorados; y para las áreas de reserva legal, se propone adoptar el modelo conocido como taungya que se caracteriza por el uso de especies arbóreas asociadas con cultivos agrícolas (Schroth et al. 2006, Vieira et al. 2009).

En general, los dos modelos agroforestales propuestos pueden tener un impacto considerable en relación a i) la conservación y recuperación de reservas de carbono en la biomasa aérea y subterránea, aumentando la densidad de siembra de árboles, y ii) mejorando la gestión sustentable de los productos forestales no maderables, iii) la conservación de la biodiversidad a través de la restauración pasiva y rehabilitación de áreas degradadas. Las principales razones para la adopción de sistemas agroforestales en Amazonas, son principalmente de carácter ambiental que también pueden tener repercusiones en el ámbito social y económico bajo un enfoque de sustentabilidad.

\section{CONCLUSIONES}

La agroforestería representa una importante alternativa para el manejo sustentable de los bosques con el propósito de recuperar y conservar reservas de carbono a través de las principales actividades REDD+ frente a la presión generada por la agricultura itinerante en la Amazonía. Por lo tanto, se proponen como acciones REDD+ i) la rehabilitación de áreas forestales degradadas, que al mismo tiempo contribuye directamente con ii) la restauración pasiva de bosques secundarios, o también a través de iii) barbechos mejorados con la incorporación de especies agroforestales durante la sucesión controlada. Finalmente, iv) la conservación permanente de bosques primarios debería ser parte clave de la estrategia integral de conservación de carbono.

Una de las premisas más relevantes para proponer a la agroforestería como un tipo de acción REDD+, surge frente a la preocupación del incremento de las emisiones de carbono a la atmósfera y disminución del potencial de los sumideros de carbono que tienen los bosques de la Amazonía a largo plazo, después de alcanzar el equilibrio e incremento de las tasas de mortalidad de árboles. Por esta razón, se sugiere que a través de la agroforestería se podrían recuperar reservas de carbono a corto plazo con las propuestas indicadas en este estudio, del mismo modo es necesario evaluar el impacto potencial a largo plazo de los de ciclos agroforestales sucesivos sobre los posibles déficits de sumideros. En un escenario futuro optimista, se espera que las tasas anuales de conservación y alma- cenamiento del carbono obtenidas mediante el conjunto de actividades REDD+ deberían aumentar con el tiempo, principalmente a través de la reducción de la deforestación y degradación forestal, pero posiblemente también los sistemas agroforestales sucesivos podrían alcanzar su máxima acumulación de carbono con cantidades similares a las de bosques secundarios en regeneración.

Con base en el conocimiento existente sobre las ventajas de los sistemas agroforestales, aún se necesita profundizar mucho más sobre el análisis de los impactos ambientales potenciales bajo escenarios actuales y futuros en la cuenca, frente a los patrones de uso de la tierra en las diferentes unidades ecológicas existentes, que además se convertiría en una acción REDD+ clave para la planificación del manejo y conservación de las reservas de carbono a escala regional. Además de las acciones asociadas con los impactos positivos derivados de la agroforestería, no se debe descartar la integración de los diferentes tipos de manejo de bosques que permiten contribuir con las cinco actividades REDD+. Así mismo, se destaca la importancia de evaluar simultáneamente el impacto sobre los componentes ambientales y sociales relacionados con la conservación de la biodiversidad, seguridad alimentaria y medios de vida sostenibles de pobladores locales como aspectos determinantes para mantener un desarrollo sustentable en esta trascendental región tropical.

\section{REFERENCIAS}

Albrecht A, ST Kandji. 2003. Carbon sequestration in tropical Agroforestry Systems. Agriculture Ecosystems and Environment 99: 15-27.

Aragão LEOC, B Poulter, J Barlow. 2014. Environmental change and the carbon balance of Amazonian forests. Biological Reviews 89(4): 913-931. DOI: 10.1111/brv.12088.

Arroyo-Kalin M. 2012. Slash-burn-and-churn: Landscape history and crop cultivation in pre-Columbian Amazonia. Quaternary International 249: 4-18. DOI: 10.1016/j. quaint.2011.08.004.

Baker T, O. Phillips, Y Malhi, S Almeida, L Arroyo, A Di Fiore, T Erwin, N Higuchi, T Killeen, S Laurance, W Laurance, S Lewis, A Monteagudo, D Neill, P Nunez Vargas, N Pitman, N Silva, R Martinez. 2004. Increasing biomass in Amazonian forest plots. Philosophical Transactions of the Royal Society of London B 359 (1443): 353-365. DOI: 10.1098/ rstb.2003.1422.

Boucher D, S Roquemore, E Fitzhughl. 2013. Brazil's Success in Reducing Deforestation. Tropical Conservation Science 6 (3): 426-445.

Brienen RJW, OL Phillips, TR Feldpausch, E Gloor, TR Baker, J Lloyd, G Lopez-Gonzalez, A. Monteagudo-Mendoza, Y Malhi, SL Lewis, R Vásquez Martinez, M Alexiades, E Álvarez Dávila, P Alvarez-Loayza, A Andrade, LEOC Aragão, A Araujo-Murakami, EJMM Arets, L Arroyo, GA Aymard, OS Bánki, C Baraloto, J Barroso, D Bonal, RGA Boot, JLC Camargo, CV Castilho, V Chama, KJ Chao, J Chave, JA Comiskey, F Cornejo Valverde, L da Costa, EA de Oliveira, A Di Fiore, TL Erwin, S Fauset, M Forsthofer, DR Gal- 
braith, ES Grahame, N Groot, B Hérault, N Higuchi, EN Honorio Coronado, H Keeling, TJ Killeen, WF Laurance, S Laurance, J Licona, WE Magnussen, BS Marimon, BH Marimon-Junior, C Mendoza, DA Neill, EM Nogueira, P Núñez, NC Pallqui Camacho, A Parada, G Pardo-Molina, J Peacock, M Peña-Claros, GC Pickavance1, NCA Pitman, L Poorter, A Prieto, CA Quesada, F Ramírez, H RamírezAngulo, Z Restrepo, A Roopsind, A Rudas, RP Salomão, M Schwarz, N Silva, JE Silva-Espejo, M Silveira, J Stropp, J Talbot, H ter Steege, J Teran-Aguilar, J Terborgh, R Thomas-Caesar, M Toledo, M Torello-Raventos, RK Umetsu, GMF. van der Heijden, P van der Hout, IC Guimarães Vieira, SA. Vieira, E Vilanova, VA Vos, RJ Zagt. 2015. Longterm decline of the Amazon carbon sink. Nature (519): 344360. DOI: $10.1038 /$ nature 14283.

Bryant D, D Neilson, L Tangley. 1997. The last forest frontier forests: ecosystems and economies on the edge. Washington, USA. World Resources Institute. 115 p.

Cerri CEP, M Easter, K Paustian, K Killian, K Coleman, M Bernoux, P Falloon, DS Powlson, NH Batjes, E Milne, CC Cerri. 2007. Predicted soil organic carbon stocks and changes in the Brazilian Amazon between 2000 and 2030. Agriculture, Ecosystems and Environment 122: 58-72. DOI: 0.1016/j.agee.2007.01.008.

Ciais P, C Sabine, G Bala, L Bopp, V Brovkin, J Canadell, A Chhabra, R DeFries, J Galloway, M Heimann, C Jones, C Le Quéré, RB Myneni, S Piao, P Thornton. 2013. Carbon and Other Biogeochemical Cycles. In Stocker, TF, D Qin, GK Plattner, M Tignor, SK Allen, J Boschung, A Nauels, Y Xia, V Bex, PM Midgley eds. Climate Change 2013: The Physical Science Basis. Contribution of Working Group I to the Fifth Assessment Report of the Intergovernmental Panel on Climate Change. Cambridge, United Kingdom and New York. Cambridge University Press. p. 465-570.

Diniz FH, MA Hoogstra-Klein, K Kok, B Arts. 2013. Livelihood strategies in settlement projects in the Brazilian Amazon: Determining drivers and factors within the Agrarian Reform Program. Journal of Rural Studies 32: 196-207. http:// dx.doi.org/10.1016/j.jrurstud.2013.06.005.

D’Oliveira MV, EC Alvarado, JC Santos, JA Carvalho. 2011. Forest natural regeneration and biomass production after slash and burn in a seasonally dry forest in the Southern Brazilian Amazon. Forest Ecology and Management 261: 1490-1498. DOI: 10.1016/j.foreco.2011.01.014.

Espindola GM, APD Aguiar, E Pebesma, G Câmara, L Fonseca. 2012. Agricultural land use dynamics in the Brazilian Amazon based on remote sensing and census data. Applied Geography 32: 240-252. DOI: 10.1016/j.apgeog.2011.04.003.

Fauset S, MO Johnson, M Gloor, TR. Baker, A Monteagudo, RJW Brienen, TR Feldpausch, G Lopez-Gonzalez, Y Malhi, H Steege, NCA Pitman, C Baraloto, J Engel, P Pétronelli, A Andrade, JLC. Camargo, SGW Laurance, WF. Laurance, J Chave, E Allie, PN Vargas, JW Terborgh, K Ruokolainen, M Silveira, GA. Aymard, L Arroyo, D Bonal, H RamirezAngulo, A Araujo-Murakami, D Neill, B Hérault, A Dourdain, A Torres-Lezama, BS Marimon, RP. Salomão, JA Comiskey, M Réjou-Méchain, M Toledo, JC Licona, A Alarcón, A Prieto, A Rudas, PJ. van der Meer, TJ Killeen, B M Junior, L Poorter, RGA Boot, B Stergios, E Vilanova, FRC Costa, C Levis, J Schietti, P Souza, N Groot, E Arets, VC Moscoso, W Castro, EN Honorio, M Peña-Claros, C Stahl,
J Barroso, J Talbot, IC Guimarães, G van der Heijden, R Thomas, VA Vos, EC Almeida, EA Davila, LEOC Aragão, TL Erwin, PS Morandi, EA de Oliveira, MBX Valadão, RJ Zagt, P van der Hout, P Alvarez, JJ Pipoly, O Wang, M Alexiades, CE Cerón, I Huamantupa-Chuquimaco, A Di Fiore, J Peacock, NCP Camacho, RK Umetsu, P Barbosa de Camargo, RJ Burnham, R Herrera, CA Quesada, J Stropp, SA Vieira, M Steininger, CR Rodríguez, Z Restrepo, AE Muelbert1, SL Lewis1, GC Pickavance, OL Phillips. 2015. Hyperdominance in Amazonian forest carbon cycling. $\mathrm{Na}$ ture Communications 6: 6857. DOI: 10.1038/ncomms7857.

Feldpausch TR, MA Rondon, ECM Fernandes, SJ Riha, E Wandelli. 2004. Carbon and Nutrient Accumulation in Secondary Forests Regenerating on Pastures in Central Amazonia. Ecological Applications 14 (4): S164-S176. http:// dx.doi.org/10.1890/01-6015.

Gatti LV, M Gloor, JB Miller, CE Doughty, Y Malhi, LG Domingues, LS Basso, A Martinewski, CSC Correia, VF Borges, S Freitas, R Braz, LO Anderson, H Rocha, J Grace, OL Phillips, J Lloyd. 2014. Drought sensitivity of Amazonian carbon balance revealed by atmospheric measurements. Nature 506: 76-86. DOI: 10.1038/nature12957.

INPE (Instituto Nacional de Pesquisas Espaciais, BR). 2013. Projecto Prodes. Consulted 01 Dec. 2013. Available in http://www.obt.inpe.br/prodes/index.php.

Kapos V, WA Kurz, T Gardner. 2012. Impacts of forest and land management on biodiversity and carbon. In Parrota JA, C Wildburger, S Mansourian eds. Understanding Relationships between Biodiversity, Carbon, Forests and People: The Key to Achieving REDD+ Objectives. Viena, Austria. A global assessment report IUFRO. p 53-78.

Lal R. 2008. Carbon sequestration. Philosophical Transactions of the Royal Society of London 363: 815-830. DOI: 10.1098/ rstb.2007.2185.

Laurance WF, MA Cochrane, S Bergen, PM Fearnside, P Delamonica, C Barber, S D’Angelo, T Fernandes. 2001. The future of the Brazilian Amazon. Science 291: 438-439.

Laurance WF, AKM Albernaz, FM Fearnside, HL Vasconcelos, LV Ferreira. 2004. Deforestation in Amazonian. Science 21: 1109-1111.

Lawrence D. 2004. Erosion of tree diversity during 200 years of shifting cultivation in Bornean rain forest. Ecological Applications 14: 1855-1869. http://dx.doi.org/10.1890/03-5321.

Luyssaert S, ED Schulze, A Borner, A Knohl, D Hessenmoller, BE Law, P Ciais, J Grace. 2008. Old-growth forests as global carbon sinks. Nature 455: 213-215. DOI: 10.1038/ nature 07276.

Malhi Y, D Wood, TR Baker, J Wright,O. Phillips, T Cochranek, P Meir, J Chave, S Almeida, L Arroyo, N Higuchi, TJ Killeen, SG Laurance, WF Laurance, S Lewis, A Monteagudo, DA Neill, P Núñez Vargas, N Pitman, CA Quesada, R Salomão, JNM Silva, A Torres Lezama, J Terborgh, R Vásquez Martínez, B Vincet. 2006. The regional variation of aboveground live biomass in old-growth Amazonian forests. Global Change Biology 12: 1107-1138. DOI: 10.1111/j.1365-2486.2006.01120.x.

Margulis S. 2004. Causes of deforestation of the Brazilian Amazon. New York, USA. World Bank working paper No 22. 107 p.

Nair PKR, VD Nair, BM Kumar, JM Showalter. 2010. Carbon sequestration in agroforestry systems. Advances Agronomy 108: 237-307. DOI: 10.1016/S0065-2113(10)08005-3. 
Nepstad DC, BS Soares-Filho, F Merry, A Lima, P Moutinho, J Carter, M Bowman, A Cattaneo, H Rodrigues, S Schwartzman, DG McGrath, C Stickler, R Lubowski, P Piris-Cabezas, S Rivero, A Alencar, O Almeida, O Stella. 2009. The end of deforestation in the Brazilian Amazon. Science 326: 1350-1351. DOI: 10.1126/science.1182108.

Nobre CA, LDS Borma. 2009 'Tipping points' for the Amazon forest. Current Opinion Environment Sustainability 1: 2836. DOI: 10.1016/j.cosust.2009.07.003.

Pan Y, RA Birdsey, J Fang, R Houghton, P Kauppi, WA Kurz, OL Phillips, A Shvidenko, SL Lewis, JG Canadell, P Ciais, RB Jackson, SW Pacala, AD McGuire, S Piao. 2011. A large and persistent carbon sink in the world's forests. Science 333: 988-993. DOI: 10.1126/science. 1201609.

Porro R, RP Miller, MR Tito, JA Donovan, JL Vivan, R Trancoso, RF Van Kanten, JE Grijalva, BL Ramirez, AL Gonçalves 2012. Agroforestry in the Amazon Region: A Pathway for Balancing Conservation and Development. In Nair PKR, D Garrity eds. Agroforestry: The Future of Global Land Use. Advances in Agroforestry 9. p. 391-428. DOI 10.1007/97894-007-4676-3_20.

Roosevelt AC. 2013. The Amazon and the Anthropocene: 13,000 years of human influence in a tropical rainforest. Anthropocene 4: 69-87.<http://dx.doi.org/10.1016/j.ancene. 2014.05.001>.

Saatchi SS, NL Harris, S Brown, M Lefsky, ETA Mitchard, W Salas, BR Zutta, W Buermann, SL Lewis, S Hagen, S Petrova, L White, M Silman, A Morel. 2011. Benchmark map of forest carbon stocks in tropical regions across three continents. Proceedings of the National Academy of Sciences 108(24): 9899-9904. DOI: 10.1073/pnas.1019576108.

Schroth G, M Souza, A Jerozolimski. 2006. Agroforestry and the conservation of forest and biodiversity in tropical landcapes- on-site and off-site effects and synergies with environmental legistation. In Gama-Rodrigues AC, NF Barros, EF Gama-Rodrigues eds. Sistemas agroflorestais: bases científicas para o desenvolvimento sustentável. Fluminense, Brasil. Universidade do Norte. p. 67-86.

Soares-Filho BS, DC Nepstad, LM Curran, GC Cerqueira, RA Garcia, CA Ramos, E Voll, A McDonald, P Lefebvre, P
Schlesinger. 2006. Modelling conservation in the Amazon basin. Nature 440: 520-523. DOI: 10.1038/nature04389.

Soares-Filho B, P Moutinho, D Nepstad, A Anderson, H Rodrigues, R Garcia, L Dietzsch, F Merry, M Bowman, L Hissa, R Silvestrinia, C Maretti. 2010. Role of Brazilian Amazon protected areas in climate change mitigation. Proceedings of the National Academy of Sciences 107: 10821-10826. DOI: 10.1073/pnas.0913048107.

Thompson ID, J Ferreira, T Gardner, M Guariguata, LP Koh, K Okabe, Y Pan, CB Schmitt, J Tylianakis. 2012. Forest biodiversity, carbon and other ecosystem services: relationships and impacts of deforestation and forest degradation. In Parrota J, C Wildburger, S Mansourian eds. Understanding Relationships between Biodiversity, Carbon, Forests and People: The Key to Achieving REDD+ Objectives. Viena, Austria. A global assessment report IUFRO. p. 21-52.

Thompson ID, M Guariguata, V Kapos, K Okabe, CBahamondez, V Heymell, R Nasi, C Sabogal. 2013. An operational framework for defining and monitoring forest degradation. Ecology and Society 18(2): 20. <http://www.ecologyandsociety.org/vol18/ iss $2 / \operatorname{art} 20>$.

Tremblay S, M Lucotte, JP Revéret, R Davidson, F Mertens, CJ Passos, CA Romaña. 2015. Agroforestry systems as a profitable alternative to slash and burn practices in smallscale agriculture. Agroforestry Systems 89: 193-204. DOI: 10.1007/s10457-014-9753-y.

Verburg R, S Rodrigues-Filho, D Lindoso, N Debortoli, G Litre, M Bursztyn. 2014. The impact of commodity price and conservation policy scenarios on deforestation and agricultural land use in a frontier area within the Amazon. Land Use Policy 37: 14-26. <http://dx.doi.org/10.1016/j.landusepol. 2012.10.003>.

Vieira DLM, KD Holl, FM Peneireiro. 2009. Agrosucessional restoration as s strategy to facilitate tropical forest recovery. Restoration Ecology 17 (4): 451-459. DOI: 10.1111/j.1526100x.2009.00570.x.

cLegacy of fire slows carbon accumulation in Amazonian forest regrowth. Frontiers in Ecology and the Environment 3: 365-369. http://dx.doi.org/10.1890/15409295(2005)003[0365:LOFSCA]2.0.CO;2. 